\title{
Azouz au pays des Gaulois
}

Carla CALARGÉ

"Pour avoir découvert le monde à travers le langage je pris longtemps le langage pour le monde."

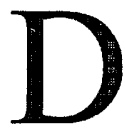

ans Le Gone du Chaâba, Azouz Begag fait une description tout en couleurs de la vie au bidonville, description qui n'est pas sans contraster avec les conditions de vie sordides qui sont celles des personnages, et que le lecteur devine au fil des pages. Pourtant, le ton n'est pas à la lamentation, ni à l'aigreur, ni même à la revendication. Bien au contraire, il est souvent comique, la lecture du roman (la première moitié surtout) se révélant parfois assez identique à celle d'une bande dessinée humoristique, celle d'Astérix en particulier. II semble, en effet, que le jeune héros perçoive sa vie comme une continuation du monde fictionnel des œ uvres qu'il lit: il serait lui-même un équivalent d'Astérix, le héros éponyme de la célèbre bande dessinée, le bidonville une version (plus ou moins) moderne du village des invincibles Gaulois et sa vie, un jeu qui combine tout cela. Car ainsi que l'affirme Rodari dans sa Grammaire de l'imagination: "Le jeu n'est pas qu'une simple reproduction 
d'impressions vécues, mais une réélaboration créatrice de celles-ci, un processus à travers lequel l'enfant combine les données de l'expérience pour construire une nouvelle réalité répondant à ses curiosités et à ses besoins" (182).

En racontant son enfance de manière ludique, Begag opère donc une double (re)création: celle d'un univers qu'il a connu et qui a disparu depuis, et celle de lui-même et de sa propre identité. C'est que, d'une part, l'identité n'est jamais séparée de ce qui a eu lieu; elle émerge au fur et à mesure que se déroule l'histoire et que l'auteur en prend conscience. Et d'autre part, l'autobiographie est tributaire de l'expérience et des lectures antérieures ainsi que l'affirme Philippe Lejeune: "[on] n'écrit jamais sans avoir lu ou entendu, et des textes en tout genre: car l'autobiographie ne se nourrit pas seulement d'autobiographie" (15). Aussi, l'identité est-elle largement déterminée par tous les mots qu'on a lus ou entendus.

Le but de cette étude est de faire une étude comparative entre le roman de Begag et l'œ uvre de Goscinny et Uderzo afin de tenter de définir les procédés au moyen desquels l'enfant Azouz arrive à créer une atmosphère rappelant l'univers fictionnel de la bande dessinée et à changer, du fait même, sa (et notre) perception de la vie au bidonville.

Le Gone du Chaâba donne à lire, en fait, une tranche de la vie du jeune Azouz Begag, celle qui s'étend du CM1 jusqu'en Sixième. La narration est prise en charge par le personnage principal qui raconte son histoire au présent de l'indicatif; la perspective narrative passe donc par un narrateur autodiégétique à travers le regard duquel le lecteur est amené à saisir la fiction. Or, dans ce roman autobiographique, s'il est tout à fait légitime de croire qu'il existe correspondance entre auteur, narrateur et personnage du fait de l'identité des noms et du recours à la première personne du singulier, il n'en demeure pas moins qu'il est pratiquement impossible de déterminer la part de connaissance rétrospective que l'auteur a de son passé puisque celui-ci s'efface parfaitement au profit de l'angle de vision de son personnage. Ainsi, le récit est-il pris en charge par Azouz enfant bien que ce soit l'adulte qui écrit. Or, "Écrire ses souvenirs, est-ce 'simplement' décrire ce qu'on voit dans sa mémoire? Tout pousse à réinventer le passé: la mémoire offre un canevas sur lequel broder" (Lejeune 37). Aussi est-il difficile de définir si c'est à Begag enfant ou adulte qu'il faudrait imputer "la responsabilité" de l'intertextualité qu'il est possible de repérer dans l'œ uvre puisque, comme dans tout récit autobiographique, des échos de lecture antérieures résonnent dans l'œ uvre.

Par ailleurs, des "allusions" réitérées à la bande dessinée Astérix le Gaulois font que la première moitié du roman baigne dans une atmosphère 
particulière qui n'est pas sans rappeler celle de la célèbre bande humoristique. Mordu de lecture, le petit Azouz est, en effet, un garçon à l'imagination fertile qui concevrait son vécu comme une continuation des histoires qu'il lit. Par un simple mécanisme d'identification, il appliquerait à sa vie certains des éléments glanés au gré de ses lectures; le monde qu'il décrit s'en trouverait alors complètement transformé. Un regard attentif sur le texte pourrait, en effet, établir des correspondances parfaites entre les personnages, les lieux, les actions et même certains éléments linguistiques des deux œ uvres, ce qui expliquerait en partie le fait que, bien que le récit de Begag ne fasse pas référence à une réalité de toute beauté, le lecteur y trouve beaucoup de plaisir et se surprend à rire de bon cœ ur.

Dès les premières pages du roman, Begag décrit une querelle qui a lieu entre les femmes du Chaâba: le ton est immédiatement donné. La scène se passe autour de "l'bomba," ou la pompe du bidonville. Or, en arabe, ce mot peut désigner aussi "la bombe" du fait que le " $p$ " n'existe pas dans cette langue et qu'il est prononcé comme un "b." Toutefois, ce point géographiquement explosif n'est nullement dangereux parce qu'au "Chaâba, on ne peut pas se haïr plus de quelques heures" (Le Gone. . . 10). Il demeure qu'au moment de "l'altercation," toutes les femmes du bidonville prennent part à la bataille et ce, à coups de poings, d'injures, de linge jeté dans la boue et de mèches de cheveux arrachées... On croirait regarder une planche d'Astérix, le Devin, celle où les habitants du village gaulois se battent à cause des poissons d'Ordralphabétix (11 vignette 10).

Ces femmes corpulentes, tout en couleurs, rappellent à bien des égards les personnages féminins de la bande dessinée; décrites souvent comme vacant à leurs travaux ménagers, elles ne manquent aucune occasion de faire éclater leurs rivalités, leurs jalousies et n'hésitent pas à assener de grands coups de balais ou de serpillières ceux qui auraient le malheur de leur désobéir: "Il a dû sortir de son lit à cause des coups de balai de sa mère. .." (Le Gone... 24).

Cependant, les occupations quelque peu bruyantes des femmes du Chaâba sont loin de représenter les seuls traits caractérisant l'atmosphère "belligérante" du bidonville. Un regard attentif au vocabulaire montrerait le recours à un champ lexical assez développé de la guerre. À travers les yeux du jeune Begag, tout est perçu en termes d'expéditions, de rounds, d'attaques, d'émeutes, d'invasion... Un passage particulièrement suggestif serait celui de "l'expédition contre les vendeuses de charme" (50), dirigée par la Louise. Begag emprunte le vocabulaire de la BD lorsqu'il 
s'agit de désigner la "compagnie," "la horde" ou "la meute" des femmes, commandée par la vieille qui fume des "gauloises." Face à ce déferlement anarchique et indiscipliné de masses fortes et inquiétantes, "les putes se rapprochent les unes des autres, pour se sécuriser" (51). Elles ont alors le même mouvement de repli que les soldats romains prenant la position carrée censée les protéger de l'attaque des Gaulois. Plus tard, lors de "l'opération commando" (68) menée par Rabah, les "ennemis" vont être obligés de capituler suite au "bombardement" intensif auquel les soumettent nos "héros." Un "impôt" (71) sera régulièrement encaissé par les vainqueurs qui garantiront alors la paix des environs et le droit de passage... Une fois la guerre finie, la communauté se réunit autour d'un café pour écouter la Louise qui raconte des histoires. C'est le soir dans le roman, mais on peut facilement imaginer la vignette finale d'Astérix: alors que l'odeur de la Chorba flotte dans les airs, le barde Assuranstourix est remplacé par le poste radio posé par terre. Le calme revient dans ce petit monde qui vit selon son propre rythme et ses propres lois: une grande famille incarnant une profonde joie de vivre et une gaieté insouciante que la civilisation n'a pas encore corrompues...

C'est que le Chaâba existe en marge de la ville, de la civilisation, mais aussi de la vie. Village "encore barbare," il a résisté jusque-là à tout effet civilisateur et continue de mener tranquillement sa vie, comme si les temps n'avaient pas changé. Ce fait pourrait faire écho à la phrase d'ouverture d'Astérix: "Nous sommes en 50 avant Jésus-Christ. Toute la Gaule est occupée par les Romains. ... Toute? Non! Un village peuplé d'irréductibles Gaulois résiste encore et toujours à l'envahisseur."

Il ne serait pas futile dès lors d'examiner les lieux tels que décrits par le texte: "Jorsqu'on franchit la grande porte en bois de l'entrée principale, on se croirait dans une menuiserie. Des baraquements ont poussé côté jardin, en face de la maison" (11). Tout n'est donc que "géométrie désordonnée" et baraques anarchiques qui s'agglutinent les unes aux autres. L'odeur du bois qui distingue le Chaâba, les constructions précaires auxquelles l'électricité n'est pas parvenue et qui s'éclairent avec les moyens du bord ainsi que la proximité de la forêt, font que le bidonville peut être facilement comparé au village d'Astérix, le seul, dans toute la Gaule, dont la civilisation romaine n'ait pas encore franchi "l'enceinte." La forêt représente d'ailleurs, pour le petit Azouz et ses amis, le lieu privilégié où peuvent avoir lieu toutes les aventures possibles, la chasse au sanglier par exemple. Azouz y va avec Hacène, le cousin qui ne brille pas par son intelligence (un autre Obélix). Affamé, celui-ci mange le casse-croûte que lui offre le héros "comme s'il s'acharn[e] sur la carcasse d'un sanglier 
tout juste abattu dans la forêt sauvage" (34). Pour le jeune Begag, les frontières du jeu sont, en effet, sans cesse repoussées plus loin de sorte que tout ce qu'il vit se transforme en épisodes divers d'une bande dessinée dont il serait le personnage principal: "Quand les choses," dit Dewey, "deviennent des signes et acquièrent la capacité d'en représenter d'autres, le jeu se transforme de simple exubérance physique en une activité qui comporte l'intervention d'un facteur mental" (Dewey, cité par Rodari 172).

Grâce à son intelligence et à son astuce, Azouz serait donc un autre Astérix. Ce qui ne manque pas de lui attirer, parfois, certains tracas; comme le jour où il est accusé de trahison et d'espionnage vu qu'il n'accepte pas d'agir aussi bêtement que les autres, ce qui l'exclut temporairement du groupe: "à présent on me soupçonne d'être infidèle" (107). Passage à comparer avec la planche du Devin où tous les habitants choisissent de croire le charlatan. Seul Astérix est alors capable de deviner la vérité (26).

Bouzid, quant à lui, serait incontestablement l'équivalent d'Abraracourcix, le chef du village gaulois, vu qu'il commande non seulement "la horde" sauvage des enfants, mais aussi tous les membres de la communauté. "Majestueux, courageux, ombrageux, ... [il] ne craint qu'une chose: c'est que le ciel lui tombe sur la tête." Autrement dit, Bouzid ne craint qu'Allah et s'efforce tout le temps d'être conforme aux préceptes de l'Islam: "Il faut toujours dire quelque chose à Allah quoi qu'on fasse!" s'exclame Béni, se moquant de son père (Béni ou le paradis privé 149). Les superstitions des Gaulois peuvent, par ailleurs, être comparées aux croyances de la famille d'Azouz aux djnouns et au mauvais œil.

Monsieur Grand, le Maître, quant à lui, représenterait Jules César. En effet, selon le dictionnaire, le mot "maître" est issu du latin magister, "chef, maître," lui-même issu de magis "plus" et désigne celui qui a le pouvoir, l'autorité, et qui exerce une domination (Dictionnaire de la langue française 1171). À travers ce personnage donc, c'est toute la civilisation française qui se profile. M. Grand apprend aux enfants non seulement la langue et les sciences mais aussi la morale, l'hygiène, la politesse et tout ce qu'il faut savoir pour devenir un bon citoyen, un parfait intégré. Il est important de noter toute l'admiration que le héros porte à son enseignant de sorte qu'il admet à un moment de l'histoire qu'il "lui obéit au doigt et l'œ il" (107). Cette attitude de la part d'un Algérien n'est pas sans être problématique. Elle rappellerait toutefois certains épisodes d'Astérix où celui-ci est obligé de composer avec César et parfois même de lui assurer la victoire afin de parvenir à ses fins. César est d'ailleurs toujours représenté comme un personnage altier qui impose 
le respect. Même le rapport des tailles, entre Astérix et lui, peut être comparé à celui qui existe entre Azouz et son enseignant. L'épisode de l'insubordination de Moussaoui est à rapprocher de celui du centurion Caius Bonus qui cherche à devenir un nouveau César (Astérix le Gaulois 27).

Moussaoui refuse d'abord de reconnaître l'autorité du maître et de lui obéir. Très vite, son attitude dégénère en "une rébellion caractérisée" ou en "coup d'État des Arabes du fond de la classe" (104). Il a d'ailleurs ses supporters que Azouz compare à une "garde impériale." Dans ce conflit ouvert, Azouz n'aidera pas les insurgés; tout comme Astérix, il fera en sorte que ce soit Jules César/M. Grand qui ait la victoire à la fin de l'histoire.

Un autre personnage assez intrigant serait celui de la Louise qui, respectée par les hommes, obéie par les femmes, est adorée par les enfants à qui elle offre, tous les jeudis, du lait au chocolat. Ce personnage condense en lui seul une somme de caractères romanesques fantastiques du fait que "Dans le jeu, comme dans le rêve, l'imagination condense les images à une vitesse fulgurante" (Rodari 177). La Louise est d'abord l'équivalent du druide Panoramix grâce à sa "potion magique" par laquelle elle récompense les enfants qu'elle choisit. Ainsi, parce qu'il ne l'a pas prévenue de l'arrivée d'un camion d'ordures, Rabah-Obélix ne pourra pas jouir de ce privilège et se contentera de bouder la petite femme à la chevelure peu fournie (détail physique qui correspond à celui du druide). Même le nom du mari de la Louise rappelle, par les phonèmes qu'il contient, "le gui" que Panoramix passe son temps à cueillir dans la forêt. Mais la Louise recoupe en elle aussi une autre image, non moins merveilleuse, puisque Begag la nomme "Louisa Baba" et qu'il compare sa maison tantôt à un château, tantôt à une caverne où elle invite régulièrement quarante gones qu'elle élit — suprême distinction! - constituant ainsi le "comité" censé pouvoir lui rendre visite et jouir des trésors de son hospitalité. Le nom de Louise lui-même n'est pas fortuit puisque, dans un autre roman, Bégag écrit: "une valise bourrée de 'louises' d'or" (Béni... 76). Marginalisée par la société française, la vieille dame revêt, dans l'imagination de l'enfant Azouz, des caractéristiques qui la transforment en un personnage fabuleux, digne des Mille et une nuits.

Tout "change" donc grâce à l'imagination fertile de notre héros qui inverserait sans cesse la réalité sordide en un conte merveilleux. Pourtant ceci n'aurait pas été possible si Begag ne se servait pas "des mots" adéquats. Ces mots opèrent, en effet, à deux niveaux: le premier serait celui, explicite, où ils sont pris exactement tels qu'ils sont utilisés dans la 
bande dessinée. Nous citons certes les mots appartenant aux champs lexicaux de la guerre et des expéditions, mais aussi des expressions-clichés d'Astérix: "Ils sont vraiment fous ces Romains" (19), des allusions narquoises à des personnages historiques: "Nous sommes tous descendants de Vercingétorix!" (62) ou au modèle gaulois: "nous sommes tous descendants des Gaulois ... et tant pis si chez moi nous n'avons pas les mêmes moustaches" (62). Le deuxième niveau, implicite, serait celui qui se sert des mêmes procédés linguistiques, typologiques et stylistiques utilisés par Goscigny et Uderzo et ce, dans le but de créer un effet comique. Ainsi, nous pouvons rapprocher, par exemple, la phrase "Et la voisine patiente toujours, elle pati... non, elle ne patiente plus" (8) du passage où les Romains combattant les Bretons finissent par perdre patience face au sang-froid de ces derniers (Astérix chez les Bretons 6, vignettes 4-8). D'ailleurs, la langue même des Bretons de la bande dessinée, antéposant sans cesse l'adjectif au nom, rappelle les diverses "variations" que font subir au français les "immigrés et leurs familles."

Un autre moyen serait de tenter de reproduire les effets produits par les vignettes peignant des sentiments extrêmes (colère, honte, embarras...) au moyen de signes typographiques et/ou de la description d'un trait physique particulièrement exagéré. La surprise du maître sera par exemple décrite en ces termes: "De quoi??! fait-il, les yeux grands ouverts de stupéfaction" (98). L'effet de surprise est peint presque de la même manière dans la bande dessinée (Astérix légionnaire 28, vignettes 7-9).

Mais le moyen linguistique le plus utilisé serait peut-être celui qui "distord" la langue phonétiquement. Dans Astérix par exemple, les noms propres des guerriers du village sont formés, en général, à partir de noms communs ou d'adjectifs finissant initialement par le suffixe "ique" que l'auteur transforme en "ix." Aussi n'est-il pas surprenant de trouver, dans Le Gone du Chaâba, des mécanismes de distorsion phonétique opérant selon des lois ressemblantes et produisant des formes phonétiquement décentrées telles finiane (fainéant), bouariane (bon à rien), La tilifiziou (la télévision)... Ce phénomène de décentrement phonétique donne en même temps lieu à un autre phénomène de décentrement graphique. L'auteur propose d'ailleurs, à la fin du roman, un "guide de la phraséologie bouzidienne" au début duquel il explique les règles du jeu de la déformation langagière (241). Car lorsqu'il s'agit de phrases françaises "passées au crible de la phonologie de l'arabe" (Sourdot 111 ), et retranscrites comme telles, le résultat rappelle, immanquablement, les passages des bandes dessinées où les auteurs reproduisent un accent étranger (le parler petitnègre par exemple). Ainsi Begag s'ingénie-t-il à multiplier, à travers son 
œ uvre, ces faits de langue narquois dont l'effet est loin de se réduire à créer une atmosphère réaliste. L'auteur ne pousse-t-il pas l'espièglerie jusqu'à opérer une nouvelle "distorsion" sur le nom d'Astérix: dans Les Voleurs d'écriture, Vincent s'écrie: "On voulait lire des Astérisques!" (79). Tout passerait donc par le biais de la langue comme semble le suggérer le jeu sur l'orthographe du mot langoureux qui devient "langue-oureux" (33). Le'geste "langue-oureux" qui renvoie, dans le roman, au fait de tirer la langue comme grimace moqueuse, Begag y a recours assez souvent, et d'abord pour ce qui est de la langue française elle-même.

Toutefois, tout comme le petit village gaulois, le Chaâba ne peut éternellement résister aux assauts de la civilisation: un jour vient où l'on doit déposer les armes et permettre à Rome d'occuper toute la Gaule. Il n'est certes pas fait mention d'un tel moment dans l'œ uvre de Goscinny et Uderzo. Dans celle de Begag, en revanche, quitter le Chaâba devient une nécessité et une évidence dès la deuxième moitié du roman. Cet épisode qui constitue "un tournant de vie" pour le narrateur correspond à ce que Lejeune appelle une "césure médiane" (105-08). Trois faits symboliques préparent et annoncent ce moment décisif de la vie du jeune Azouz: la circoncision d'abord, "le scandale de la boucherie clandestine" (136) ensuite, et la découverte de Baudelaire enfin.

Située presque au milieu du roman, la cérémonie de la circoncision d'Azouz signe la fin de l'enfance de ce dernier du fait que la circoncision "répète la section du cordon ombilical pratiquée à la naissance de l'enfant et symbolise une nouvelle naissance, c'est-à-dire l'accès à une nouvelle phase de la vie" (Chevalier, Gheerbrant 258). Le passage qui suit cet épisode est d'ailleurs éloquent: Hacène et Azouz convainquent Saïda de se faire "enculer" par eux afin d'imiter leurs parents (120-21). La nature même du jeu a changé, on reconnait certes encore la candeur de l'enfance, mais les gones commencent désormais à s'intéresser à des jeux moins innocents.

Jouer aux grands ne va pas se révéler sans conséquences: croyant faire le nécessaire pour devenir un citoyen respectable, Azouz va indiquer aux policiers, venus enquêter sur le lieu de la boucherie clandestine, l'emplacement de celle-ci. Cette dénonciation va déclencher une série de "malheurs" dont la convocation de Bouzid et de son frère au commissariat n'est pas le moindre. En effet, Zidouma, la belle-sœ ur de Bouzid, va s'insurger contre l'autorité de ce dernier. Ce faisant, elle va mettre en question les fondements même du Chaâba et, à travers cette attitude, l'obéissance aveugle aux coutumes algériennes. Ainsi non seulement il y a "insurrection" au Chaâba, mais aussi — et surtout — c'est une femme 
qui la crée. Dès lors, le bidonville ne peut plus exister: le fait que Zidouma ait eu le courage de contester l'autorité du chef prouve bien que l'Algérie est bien loin et que, désormais, c'est en France que l'on se trouve; l'état de flottement temporaire entre deux pays et entre deux cultures, symbolisé par la vie au Chaâba, doit prendre fin: le Chaâba commence donc à se vider de ses habitants et à "mourir" lentement. C'est ce qui explique d'ailleurs l'atmosphère lugubre et triste qui s'installe dans le bidonville: Begag "recovers, through memory, the lost paradise that was the 'chaâba,' a place in which the village life of Algeria was recreated by immigrants to France" (Lay-Chenchabi 2).

Parallèlement à ces changements, le héros découvre Baudelaire à l'école. Certains indices intertextuels peuvent suggérer que l'auteur est un admirateur de la poésie baudelairienne dont il cite un quatrain en exergue de son roman Les Chiens aussi. Cette $œ$ uvre où le narrateur principal est un chien/immigré (ou un chien d'immigré) est dominée par l'image de l'Albatros qui y symboliserait l'âme de l'artiste, du génie, mais aussi la liberté et la possibilité d'une élévation vers des sphères inconnues, d'un envol "au pays du Bonheur" (125). Toujours est-il qu'avec Baudelaire une période est définitivement révolue: celle de l'enfance insouciante au Chaâba.

Il demeure cependant que Baudelaire, $M$. Grand ou l'école en général représenteraient, pour Azouz, la possibilité de se trouver et de se mettre en contact avec la civilisation, la culture et la langue françaises. Dans Béni ou le Paradis privé le héros tombe amoureux d'une jeune fille de sa classe qui s'appelle France. On devine bien que ce prénom n'est pas arbitraire. De même, dans Le Gone du Chaâba, Azouz est captivé par ce pays où il est né et qui, jusque-là, ne l'a pas complètement accepté. Pour se faire aimer, il a compris qu'il ne peut rester "derrière," au fond de la classe, et qu'il doit se démarquer des autres Arabes bien que cette attitude lui cause des sentiments de honte et de culpabilité: "J'ai terriblement honte des accusations que m'ont portées mes compatriotes parce qu'elles étaient vraies" (107). Mais cela ne l'empêchera pas, plus tard, d'affirmer qu'il est Juif lorsqu'il sera sommé de faire un choix. Pouvoir changer d'identité et de personnalité selon les situations et le besoin est d'ailleurs le rêve de Béni:

Je voulais lui dire qu'un comédien avait le magique avantage d'être plusieurs gens à la fois, avec le choix de se cacher dans la peau de l'un d'eux, . . comédien pour faire croire qu'on n'est pas celui qu'on est en réalité, et vice versa, personne ne me comprendrait plus et ce serait tant mieux comme ça car on ne 
serait plus assuré de rien, bien fait! Un monde fait de comédiens dans lequel on ne saurait pas si monsieur Untel s'écrit avec un $\mathrm{U}$ majuscule ou un I. ... (74)

Tel serait aussi le rêve du jeune Azouz. Brouiller les cartes, être à la fois tout le monde et personne, écrire son nom tantôt avec un $\mathrm{U}$ et tantôt avec un I parce que cela se prononce de la même manière. On le devine, l'aventure du jeune héros passe d'abord par les mots, par la langue et par les livres. Le narrateur des Voleurs d'écritures ne parle-t-il pas d'ailleurs de "l'air livre" (85)? N'affirme-t-il pas que "Si t'as vraiment vu de l'or, il est forcément dans les livres" (67)? Seuls les mots qui racontent de "fausses" histoires peuvent rendre l'histoire réelle supportable, seule l'imagination qui permet de voir, dans sa tête, des choses différentes peut transformer le vécu en quelque chose de plus doux. Dans Les Chiens aussi, le narrateur/ chien vole un livre du cartable d'un enfant, le baptise Merlin, Pinpin et Malou et "[s'invente] une lecture" (71) qui le fait rêver. Il est facile de deviner l'allusion à Merlin l'enchanteur et à Tintin et Milou: dans ces œ uvres où les bons sont récompensés et où les méchants sont châtiés, la vie est une aventure passionnante et le monde un espace à conquérir. Il serait significatif alors de noter que le héros/chien s'appelle "César" dans la fiction. Doit-on le mettre en rapport avec Jules César? Il s'agit certes d'un conquérant capable de mobiliser la foule pour une cause juste.

Toujours est-il que c'est à travers les livres et la langue que se joue l'essentiel de l'aventure "azouzienne" et ce, à deux niveaux: celui d'abord qui permet de recréer le passé grâce au travail de la mémoire (et ce faisant, créer son identité), et celui ensuite, qui permet à l'enfant Azouz de réussir ses premiers pas d'intégration dans la société française du fait que son parcours "peut être lu comme celui de l'intégration d'un enfant immigré qui, très tôt, a pris conscience que la réussite de cette intégration passait par la maîtrise de la langue du pays d'adoption" (Sourdot 109).

Ainsi donc, son entrée définitive dans la langue française représente, pour le jeune Azouz, la pré-condition de devenir conscient de lui-même comme entité distincte d'une part, et d'autre part, comme citoyen français, identitairement hybride. A ce niveau, l'identification avec Astérix revêt une autre dimension, car ainsi que l'affirme Philippe Forest, "Astérix correspond tellement à l'image consensuelle que les Français se font d'eux-mêmes qu'on a pu lui assigner toutes les places sur l'échiquier idéologique national" (97). Astérix est donc le personnage auquel tout Français peut s'identifier, mais aussi, il est celui qui a pu défier les superpuissances et se tailler (grâce à la potion magique) un fief libre à l'intérieur de l'empire romain. 
Par ailleurs, en envahissant une grande partie de l'Europe, Rome/ César impose le latin comme langue officielle dans les "colonies romaines." Cette langue, mise en contact avec celles des indigènes se transforme au cours des siècles: "vulgarisé" d'abord, le latin donne naissance, par la suite, à une série d'autres langues — dont le français — qui se réclament aujourd'hui d'une racine commune. Or, le français "distordu" de la littérature beure n'est-il pas en train de répéter ce même schéma transformationnel? Celui justement où un "chirurgien" peut être pris pour un "chirurchien" grâce aux pouvoirs créateurs et à l'imagination d'un "magichien" (Les chiens aussi)!!? Il est certes trop tôt pour pouvoir le dire. Toujours est-il que nous pouvons avancer que, tout comme César a dû compter avec le petit village gaulois, la France aujourd'hui ne peut ignorer les immigrés et leurs familles: elle s'interroge. . (Cf. Astérix le Gaulois 5, vignette 6)

\section{University of Iowa}

\section{Works Cited}

Begag, Azouz. Le Gone du Chaâba. Paris: Seuil, col. Points virgule, 1986. —. Béni ou le Paradis privé. Paris: Seuil, col. Points Virgule, 1989.

-. et Catherine LOUIS. Les Voleurs d'écriture. Paris: Seuil, col. Petit Point, 1990.

—. Les Chiens aussi. Paris : Seuil, 1995.

Chevalier, Jean et Alain Gheerbrant. Dictionnaire des symboles. Paris:

Robert Laffont et Éditions Jupiter, $7^{\mathrm{e}}$ édition, 1987.

Forest, Philippe. "Astérix" in Dictionnaire des mythes d'aujourd'hui. sous la direction de Pierre

Brunel, avec la collaboration de Frédéric Mancier et Matthieu Letourneux.

Paris: Editions du Rocher, 1999.

Goscinny, René, Uderzo, Albert. Astérix chez les Bretons. Paris: Hachette, 1999.

-. Astérix légionnaire. Paris: Hachette, 1999.

- Astérix le Gaulois. Paris: Hachette, 1999.

- Astérix, le Devin. Paris: Hachette, 1989.

Lay-Chenchabi, Kathryn. "Writing for their lives: three Beur writers discover themselves." Mots Pluriels 17 (Avril 2001) <http:// www.cm.refer.org/motspluriels/MP1701klc.html>.

Lejeune, Philippe. Les Brouillons de Soi. Paris: Seuil, 1998. 
Rodari, Gianni. Grammaire de l'imagination. Paris: Rue du Monde, 1997. Sourdot, Marc. "Un héros recentré: Le Gone du Chaâba d'Azouz Begag" in L'Écriture décentrée. La langue de l'autre dans le roman contemporain. Ed. Michel Laronde. Paris: L'Harmattan, 1996. 